\title{
A Framework for Self-Management of Hybrid Wireless Networks Using Autonomic Computing Principles
}

\author{
Chong Shen, Dirk Pesch \\ ${ }^{1}$ Cork Institute of Technology \\ Centre for Adaptive Wireless \\ Bishopstown, Cork, Ireland \\ $\{$ cshen, dpesch\}@cit.ie
}

\author{
12 James Irvine \\ ${ }^{2}$ University of Strathclyde \\ Mobile Communications Group \\ Glasgow, Scotland, UK \\ j.m.irvine@ieee.org
}

\begin{abstract}
The dramatic increase in the number of mobile subscribers has put a significant resource and service provisioning strain on current cellular networks in particular in terms of multimedia and high-data rate service provision. Hybrid wireless networks, which is a novel scalable and adaptive wireless network architecture utilizing a mixture of cellular and ad hoc multi-hop routing, facilitates cellular network design with small cell systems without having to wire a large number of base stations into a core network. However, this new network design has drawbacks in terms of routing complexity, radio resource heterogeneity and network infrastructure design growth. Traditional centralised system administration methods become too inflexible to result in an manageable cellular system. A recently introduced concept - Autonomic Computing, based on stimulation from biological systems, may provide a remedy to the state of unmanageability. The Autonomic computing paradigm, recently coined autonomic communications, when applied to communication systems and networks, enables self-management, which is composed of self-protecting, self-healing, self-configuring and selfoptimizing components. This self-management is not necessarily novel as technologies such as neural networks, fuzzy logic, genetic algorithms and evolutional methods have already been proposed to facilitate limited self-configuration and self-optimization when embedded with cellular and ad hoc networks. However, a structure or framework has been lacking so far. In this paper we propose an architecture for a framework of autonomic computing based on policies for a hybrid wireless network and investigate the merits of each of its functions.
\end{abstract}

\section{Introduction}

Supporting both high data rate and ubiquitous coverage in wireless networks poses fundamental difficulties to either transmitter power consumption or wireless networking costs. An architectural approach to remedy this problem is called hybrid networks [1]. A number of problems arise from this new wireless network architecture, in particular the large number of access points or base stations in the system cannot be efficiently managed and configured through a centralized interface anymore as is the case in current cellular networks. A flexible, self-management approach for radio resource management (RRM), multi-hop radio relaying, and routing needs to be developed. The new paradigm of autonomic computing (AC) [2] may provide the approach to solve these issues. The paradigm of $\mathrm{AC}$ was proposed by IBM [3] for enabling self-optimizing, self-protecting, self-configuration and self-healing systems. Motivated by $\mathrm{AC}$, we propose an intelligent infrastructure, which is composed of distributed mobiles stations, routing stations and base stations, based on $\mathrm{AC}$ that meets the demands for high bandwidth multimedia applications with ubiquitous coverage. Such a system presents a first step toward autonomic communications.

The next section discusses the background behind AC, proposed applications and implementations. A review of hybrid cellular and ad hoc networks is presented briefly in Section 3. Section 4 investigates how we can meet AC requirements with our proposed framework embedded in the hybrid wireless system. Section 5 describes the related work, which has been done previously and finally in Section 6 we draw a conclusion and elaborate on our future effort.

\section{Autonomic Computing}

When overlapping connections are growing, when system dependencies are unclear, and when interacting ap- 
plications are conflicting, normal decision-making and response mechanisms may not be able to provide a solution anymore. Without new approaches, more complexity cannot be achieved. AC means embedding intelligent control into the system infrastructure itself (both hardware and software) in order to automate everything in a self-managing manner; new components integrate as effortlessly as a new cell seamlessly establishes itself in the massively complex human body [2]. Therefore, our approach is motivated by $\mathrm{AC}$, which has interrelated capabilities of self-optimizing, self-protecting, self-configuration and self-healing.

The Autonomic architecture is composed of interactive collections of autonomic elements (AE) [10], Figure 1, AEs manage both their relationships with other AEs and internal behavior according to established policies. An AE typically consists of one or more managed elements coupled with a single autonomic manager that controls and represents them [2]. The Autonomic manager is responsible for monitoring and managing the knowledge-based processes such as monitor, analyze, plan and execute in managed elements. AEs, in computer systems for example, function at many levels from disk drivers to workstations to entire systems. It is envisaged that as AEs with self-managing properties become general components of a computer system, they will merge into a super powerful intelligent system. To achieve self-manageability, W. Trumler et all propose a Smart Doorplate into AC for a completely distributed application [4], where self-configuration is achieved by reducing manual operations to the absolute minimum. S. Whiteson and P. Stone investigate adaptive network routing and scheduling toward AC [5]. The results show clearly that machine learning methods offer a significant advantage in self-optimizing the performance of complicated networks. Yu-Han Chang et al. provide a reinforcement learning approach based mobilized ad hoc network within the context of AC [6] where self-optimizing and adaptive learning (selfconfiguration) make this an adaptive algorithm for controlling movement and routing for ad hoc networks.

\section{Hybrid Wireless Networks}

A hybrid wireless network architecture is a network that provides dedicated relay stations and embedded host-cumrelay stations with mobile terminals; further, the architectures can be divided into single-mode hybrid, where mobile terminals only perform a single hop, and multi-mode hybrid, where either multi-hop or single-hop happens in mobile terminals. The recent attempts at throughput enhancement in hybrid networks include multi-hop cellular network (MCN) [11], multi-power architecture for cellular networks (MuPAC) [7], integrated cellular and ad hoc relaying system (iCAR) [7], self-organizing packet radio networks with overlay (SOPRANO) [8] etc. (Figure 2). In MCN [11], av-

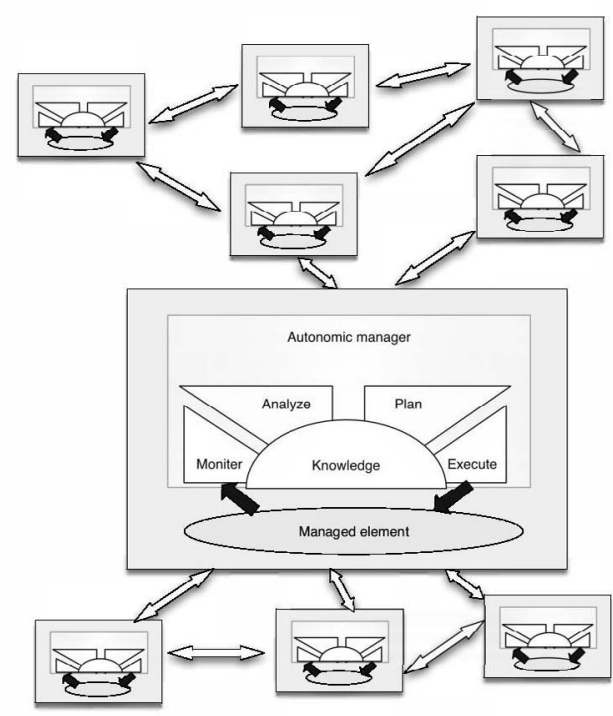

\section{Figure 1. Structure of an autonomic element. Elements interact with other Elements with autonomic managers}

erage path length for a path between source and destination increases with $k$ ( $k$ is reuse factor) and the number of simultaneous transmissions possible increases as $k^{2}$. Thus, throughput could be expected to increase linearly with $k$, but unfortunately the actual gain will be lower due to numerous routing overhead and possible absence of relay mobile terminals. iCAR [7] is not difficult to evolve from the existing cellular networks and enable the network to achieve theoretical capacity improvement through adaptive traffic load balancing. Excessive bandwidth available in surrounding cells could be borrowed to the congested cell through dedicated ad hoc relay modes named primary, secondary and cascaded relaying. SOPRANO [8] is a novel scalable architecture that assumes the use of asynchronous CDMA with lots of spreading codes to support high data rate Internet and multimedia traffic. The general idea of SOPRANO is not much different to iCAR other than IP network support and cross network connection.

Although advanced technologies make single-cumrelayed complicated mobile stations (individual moving users) feasible, recalculating and reconfiguring mobile terminal topology in infrastructure such as MCN and MuPAC brings intolerable management cost. Therefore, adaptive and scalable wireless network architectures with dedicated relay stations are more suitable and cost-effective. In Figure 3, a hybrid network Structure with dedicated relay stations is presented. Base Stations (BS), Relay Station routers (RO), and Terminals (TE) are distributed uniformly in the 


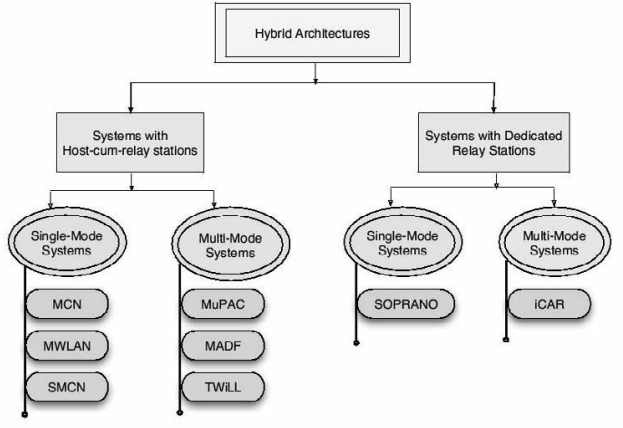

Figure 2. Classification of Hybrid Architectures

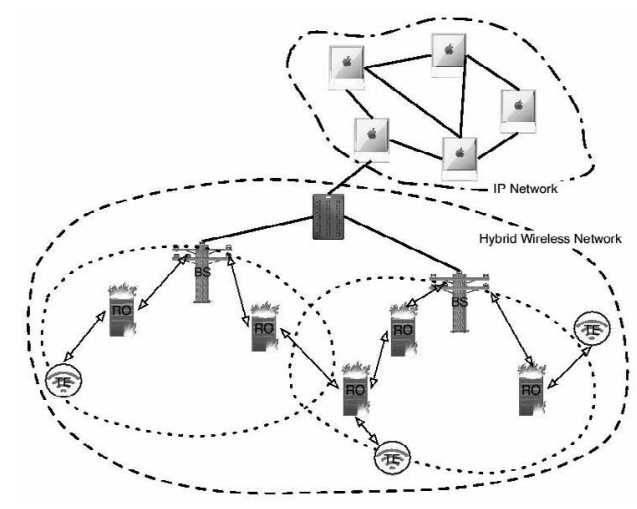

Figure 3. Hybrid Wireless Network with Dedicated Relaying Stations

network. TE in a cell might transmit to a BS in another cell because the current cell is a hot spot (too much traffic in one cell).

Time division duplex code division multiple access (TDD-CDMA) is the multiple access technology we assume for investigation of $\mathrm{AC}$ based management in hybrid networks. TDD-CDMA is an attractive multiple access technology for hybrid networks as it allows radio resources to process network traffic in both directions, uplink and downlink (The drawback of frequency division duplex (FDD) based standard is, with asymmetric loads of downlink and uplink, portions of the spectrum are occupied but not used). Furthermore, it offers a smooth and seamless way of upgrading existing GSM networks towards 3G networks and services[13]. Further advantages of TDD-CDMA based hybrid networks are that smart antennas permit focusing of transmission beams and minimizing cell interference and increasing transmission capacity. Joint detection eliminates the multiple access interference typically associated with multi-user access through parallel processing of individual traffic streams. Terminal synchronization improves the uplink quality by precisely tuning the transmission timing of each individual terminal with respect to its base station[14]. Soft handover of CDMA where the mobile stations connects with two or more base stations can also be used in TDDCDMA for the benefit of power efficiency.

Cellular networks with dedicated relay stations result in an increasingly large amount of base sites that share spectrum with mobiles, which becomes unmanageable with a centralized management approach. Therefore, focusing on Autonomic Computing based self-management can lead to new solutions to overcome the management problem.

\section{Self-Management}

We study AC methods for addressing the problems of hybrid wireless networks. Ideally, we require every communication layer within the hybrid network with dedicated relaying stations to include all of the abilities of selfmanagement. Each layer is treated as an $\mathrm{AE}$ and all layers are interacting atomically. Especially, a feed back AE that can train and learn from the past experience is designed as a system monitor. RRM in the MAC layer protocols and hybrid routing protocols are emphasized in our study and the other protocols are assumed accordingly.

To achieve self-management in a system, it should be both self-aware and environment-aware. Self-aware means system should know the current status of itself while environment-aware means the awareness of operating environment. Self-management is created though self-configuration, self-optimization, self-healing and selfprotecting activities.

\subsection{Self-configuration}

In a high-capacity cellular system we always find devices that compete for the same resource no matter how we try to share the available radio spectrum. Therefore, a self-configurable MAC protocol in hybrid wireless networks, which considers RRM elements such as call admission; channel allocation and handover should be given special attention. Self-configuration involves automatic incorporation of new components and automatic component adjustment to new conditions. For example, when a new call request is received by the network, hybrid systems should know which channel is best to carry signaling and data. If the current cell where the call request is received, happens too be too busy to accommodate the request, the system should know an alternative and direct the call to another, adjacent cell through dedicated routers without any manual operation. Another example is where the cell size is adjusted using cell breathing algorithms to re-configure the 
service area and redirect calls to other cells for better load balancing.

\subsection{Self-optimization}

To automatically manage hybrid wireless networks, selfoptimization is given special attention for routing protocols. Because the topology of hybrid networks can change dramatically with routers potentially appearing and disappearing from the network, congestion or link failure increases with the number of node. A good self-managed protocol for routing packets with mobility support is desirable. Selfoptimization at the system level is about automatic parameter tuning based on services. A suggested method to do this is to explore, learn and exploit, such as a self-learning circle. For example, when a route becomes congested or fails, the protocol should have mechanisms to know what happened and use an alternate path. Not only does the protocol satisfy this with routing re-selection, but it should also learn from the past experience to produce an optimum routing strategy. Therefore, adaptive code, which implements the adaptive behavior is vital to AC based routing protocol design. The predominant mechanism for implementing adaptation in routing protocols is behavioral reflection [8], which can be used to modify how protocols respond to dramatic traffic changes such as congestion, link failure and fault tolerance.

\subsection{Self-healing}

Self-healing from bugs and failures can be accomplished using components for detection, diagnosis and repair. As we know the transport layer is responsible for end-to-end error recovery and flow control. With an adaptive self-healing mechanism, the transport layer is more reliable in providing transparent transfer of data and voice traffic between two individual mobile users, thus relieving the application software from any concern with providing reliable and costeffective data transfer.

\subsection{Self-protecting}

Self-protection of systems will prevent large-scale correlated attacks or cascading failures from permanently damaging valuable information and critical system functions. It may also act proactively to mitigate reported problems. Thus, it is useful if implemented in hybrid systems network protocols design. Once the physical path is decided from the sender mobile station to a receiver mobile station, the self-protecting mechanism should protect channels from any unauthorized access and maintain overall stability.

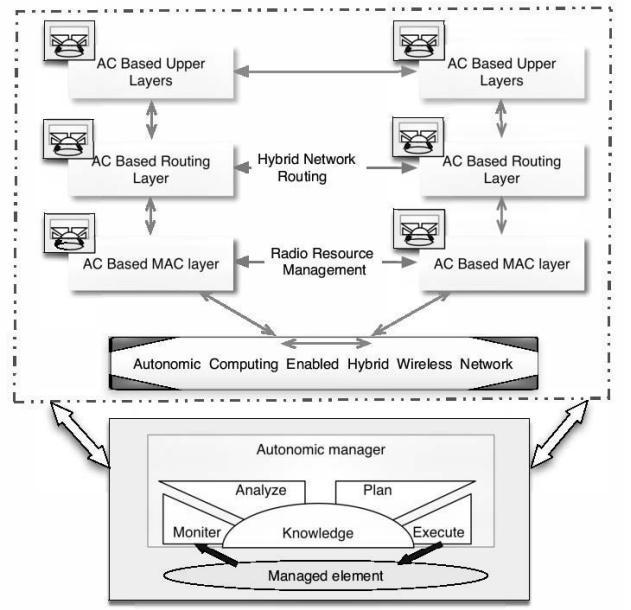

Figure 4. Autonomic Computing Based Hybrid Wireless Network

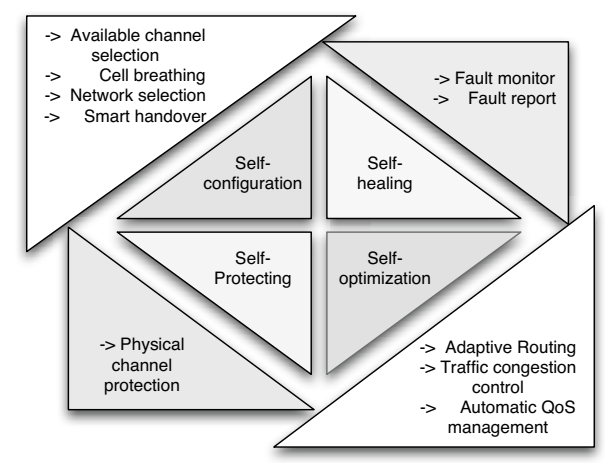

Figure 5. The Autonomic Features of AC Elemments in Hybrid Networks

\section{Using Autonomic Computing Principles}

Based on the four AC systems functions we discussed above, we propose an AC based hybrid network structure in Figure 4. We use adaptive software applications in upper layers for simplified software development and maintenance. Data packets and voice traffic is sent in a reliable environment and we assume a perfect secure network layer. A smart routing protocol, which enables self-optimization is used and in the Mac layer, radio resources are managed in a self-configurable and self-optimising manner using learning based strategies. Meanwhile, the autonomic features are mapped in Figure 5 which are available to all AC elements in a hybrid network.

In this AC based architecture, interactions between each 
layer are accomplished through interfaces[12]. Monitoring and test interfaces in $\mathrm{AE}$ is responsible for testing and inspecting services provided by other AEs. Lifecycle interfaces are in charge of determining the lifecycle state such as started and paused in an AE. Self-management policies are communicated within interfaces policy interfaced (Selfmanagement policies are discussed in the section on related work) and negotiation and binding interfaces allow services request between AEs.

Artificial intelligence (AI) techniques such as Neural Networks, Fuzzy logic and Q-learning are attractive approaches to facilitate autonomic operation in the routing layer and MAC layer as these technologies are not only suitable parts of AEs but also fulfill all of the requirements for monitoring, analysing, planning and executing. Moreover, they are knowledge based, which is a pre-requisite for autonomic operation.

The desire for automation and intelligence systems is not new. As a matter of fact, AI based self-management in cellular networks has been a major research goal for many years. What we consider new in this proposal is its overall breadth of scope including self-optimization, selfprotecting, self-configuration and self-healing. The interesting aspect is to study the collaboration between AI techniques (including machine learning methods) and hybrid systems in order to manage the inherent complexity of these systems.

\section{Example AC Research}

In this section, two main research directions related to AC infrastructure on RRM are outlined. To boost the efficiency of cellular networks, high-level self-management policies are indispensable. On the other hand, distributed RRM algorithms demonstrate greater flexibility and scalability offered by distributed computing approaches. Thus AC self-management can be realized by incorporation of distributed structures with AI methods.

\subsection{Self-Management Policies}

AC based self-management of hybrid network behavior should be represented by high-level directives to be translated into specific actions to be taken by AE based layers. Therefore, policing the behavior is of great importance.

To cover the broadest possible range of situations and contexts, three interrelated policy types should be considered[12]. In Figure 6, action policies, goal policies and unity policies are arranged from lowest level to the highest level with examples. Action policies in the lowest level are typically of the form IF (condition), THEN (action), which means AEs must execute the stated actions whenever the condition is satisfied. Goal policies specify

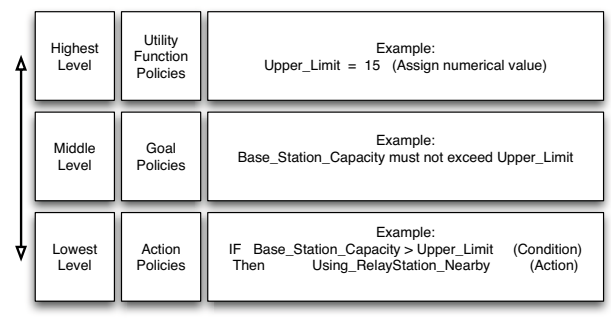

\section{Figure 6. Self-Management Polices of Hybrid Network}

the conditions to be obtained but not tell how to obtain them. Goal policies are situated at a policy level above action polices which means it has more influence than action policy because AEs can make a request to other autonomic elements without detailed knowledge. Utility functions polices are at the top level, they automatically determine the most valuable goal in any given situation, thus they have most control over the behavior of system as a whole.

Automated decisions in each of three polices are made by using machine learning approaches such as neural networks training. It is a three steps process:

- The policy server analyzes the current state and determine whether the goals are met or not.

- After information exchange through Light-weighted Directory Access Protocol (LDAP), a list of candidate rules is generated by directory services.

- The policy decision point decides the combination of rules to be invoked from amongst the list of candidate rules and finally makes the decision.

\subsection{Distributed Algorithm in MAC Layer}

Distributed dynamic channel allocation (DDCA) facilitates self-configuration at the MAC layer, where channel assignment decisions are made by individual base stations. Using intelligent decision strategies based on neural networks for example distributed channel allocation can improve on system throughput compared to centralized dynamic and fixed channel allocation where decisions are made by complex central computing systems. DDCA can therefore also achieve the objective of self-optimization. In Figure 7[15], we present results of an analysis of call blocking probabilities for three kinds of DDCA compared to centralized FCA. We can observe that the blocking performance of each of the three DDCA schemes is superior to the FCA scheme. On the other hand, the poor blocking probability of FCA schemes could be attributed to 


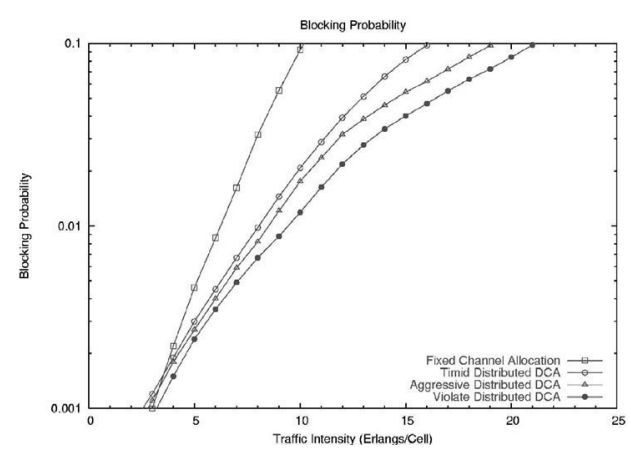

Figure 7. Call Blocking Probability

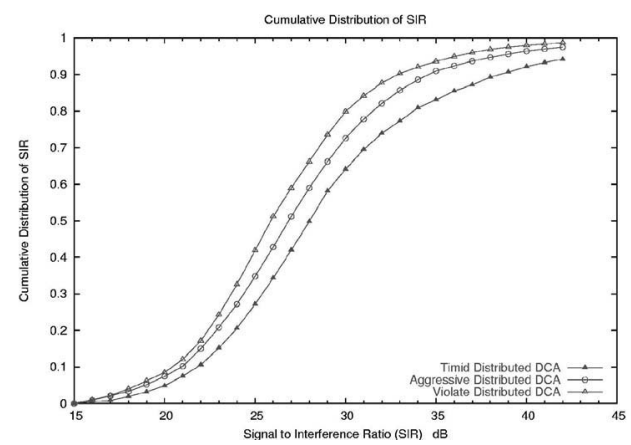

Figure 8. Cumulative Distribution of Distributed DCAs

poorer coordination and optimization of the whole systems while DDCA schemes benefit from adaptive behavior implemented through distributed decision making. In Figure 8 , the cumulative distribution function of the mean Signal to Interference Ratio (SIR) values in the network under a load of 9 Erlang/Cell and different users are spread around the target SIR values of $17 \mathrm{~dB}$ is shown.

\section{Conclusion and Future Work}

In this paper, a framework for self-management in hybrid wireless networks based on AC principles is discussed. We outlined the AC background and its requirements for self-configuration and self-optimization. We have chosen a TDD-CDMA based cellular network with a relay station architecture as the test bed of our AC based study as it lends itself to self-management. The implementation of AC within the hybrid system is presented and more detailed algorithms on AC based RRM and AC based routing are subject of our future work.

\section{References}

[1] J. Hubaux D. Nagel and B. Kieburtz, "Provision of Communication Services over Hybrid Networks," IEEE Comms magazine, Vol. 37, pp. 36-37, July 1999.

[2] J. O. Kephart and D. M. Chess, "The vision of autonomic computing," IEEE Computer, vol. 36, No.1, pp.4150, 2003.

[3] P. Horn, "Autonomic Computing: IBMs Perspective on the State of Information Technology," http://www.research.ibm.com/autonomic/, Oct. 2001.

[4] W. Trumler et all, "Smart Doorplate-Toward an Autonomic Computing," IEEE 5th Autonomic Computing Workshop, pp.42-49, 2003.

[5] S. Whiteson and P. Stone, "Toward Autonomic Computing: Adaptive Network Routing and Scheduling," IEEE, ICAC, pp.286-287, May 2004.

[6] Y. Chang, T. Ho and L. Kaelbling, "Mobilized ad-hoc networks: A reinforcement learning approach," IEEE, ICAC, pp. 240-247, May 2004.

[7] C. Murthy and B. Manoj, Ad Hoc Wireless Networks: Architectures and Protocols, PRENTICE HALL, New Jersey, 2004.

[8] A. Zadeh et all, "Self-Organizing Packet Radio Ad Hoc Networks with Overlay (SOPRANO)," IEEE, Communications Magazine, PP.149-157, June 2002.

[9] S. M. Sadjadi et all, "Generation of Self-Optimizing Wireless Network Applications," IIEEE, ICAC, pp. 3840, July 2004.

[10] IBM and autonomic computing, "An architectural blueprint for autonomic computing computer," April 2003.

[11] R. Ananthapadmanabha, et. all, "Multi-hop Cellular Networks: The Architecture and Routing Protocol," IEEE, PIMRC, vol.2, pp.78-82, Oct. 2001.

[12] S. White et all, "An architectural Approach to Autonomic Computing," IEEE, ICAC, pp.2-9, may 2004.

[13] Technical Specification, "Radio Interface Protocol Architecture," China Wireless Protocol Architecture, TS C001, V3.0.0, Oct. 1999.

[14] TD-SCDMA forum, " $3 G$ and TD-SCDMA," www.tdscdma-forum.org, June 2004.

[15] C. Shen, D. Pesch and J. Irvine, "Distributed Dynamic Channel Allocation with Fuzzy Model Selection," ITT Conference, Limerick, Ireland, Oct. 2004. 